\title{
Coupled Phosphatase and Kinase Switches Produce the Tristability Required for Long-Term Potentiation and Long- Term Depression
}

\author{
Hyun Jae Pi ${ }^{1}$ and John E. Lisman ${ }^{2}$ \\ ${ }^{1}$ Quantitative Biology Program, Department of Physics and ${ }^{2}$ Department of Biology, Brandeis University, Waltham, Massachusetts 02454
}

\begin{abstract}
Studies of long-term potentiation (LTP) and long-term depression (LTD) strongly suggest that individual synapses can be bidirectionally modified. A central question is the biochemical mechanisms that make LTP and LTD persistent. Previous theoretical models have proposed that the autophosphorylation properties of CaMKII could underlie a bistable molecular switch that maintains LTP, and there is experimental support for this mechanism. In contrast, there has been comparatively little theoretical or experimental work regarding the mechanisms that maintain LTD. Several lines of evidence indicate that LTD is not simply a reversal of previous LTP but rather involves separate biochemical reactions. These findings indicate that a minimal model of the synapse must involve a tristable system. Here, we describe a phosphatase (PP2A) switch, which together with a kinase switch form a tristable system. PP2A can be activated by a Ca ${ }^{2+}$ dependent process but can also be phosphorylated and inactivated by CaMKII. When dephosphorylated, PP2A can dephosphorylate itself. We show that these properties can lead to a persistent increase in PP2A during LTD (as reported experimentally), thus forming a phosphatase switch. We show that the coupled PP2A and CaMKII switches lead to a tristable system in which the kinase activity is high in the LTP state; the PP2A activity is high in the LTD state, and neither activity is high in the basal state. Our results provide an explanation for the recent finding that inhibition of PP2A prevents LTD induction.
\end{abstract}

Key words: synaptic plasticity; LTP; LTD; kinase; phosphatase; tristability; multistability; molecular switch

\section{Introduction}

Substantial progress has been made in understanding how synaptic activity produces bidirectional modifications of synaptic strength [long-term potentiation (LTP) and long-term depression (LTD)]. Single synapse analysis has shown that LTP and LTD occur at individual synapses (O'Connor et al., 2005; Petersen et al., 1998; Sobczyk and Svoboda, 2007). It has been further shown that LTP and LTD are associated with an increase and decrease of spine volume, respectively (Zhou et al., 2001; Matsuzaki et al., 2004). Thus, the biochemical machinery at individual synapses must be capable of at least three stable states.

A CaMKII switch has been strongly implicated in LTP. Activation of this kinase during LTP occurs by an autophosphorylation reaction at Thr286 of the $\alpha$ subunit (Fukunaga et al., 1995; Barria et al., 1997). Once this site becomes phosphorylated, the enzyme can phosphorylate itself even in the absence of $\mathrm{Ca}^{2+}$ (Miller and Kennedy, 1986). Theoretical models have shown that such autophosphorylation could sustain the autophosphorylated

Received May 23, 2008; revised 0ct. 6, 2008; accepted 0ct. 11, 2008.

This work was supported by National Institutes of Health (NIH) Grant R01 NS027337-19 and National Institute of Neurological Disorders and Stroke-NIH Grant R01 NS050944. We thank Drs. Edwin Richard, Nikolai Otmakhov, and David Brautigan (University of Virginia, Charlottesville, VA) for helpful comments on this manuscript; and Drs. Kong-Fatt Wong (Princeton University, Princeton, NJ), Irv Epstein, Anatol Zhabotinsky, and Paul Miller for useful discussions.

Correspondence should be addressed to John E. Lisman, Department of Biology, Brandeis University, Waltham, MA 02454. E-mail: lisman@brandeis.edu.

D0I:10.1523/JNEUROSCI.2348-08.2008

Copyright $\odot 2008$ Society for Neuroscience $\quad$ 0270-6474/08/2813132-07\$15.00/0 state; CaMKII could thus form a bistable switch (Lisman, 1985; Zhabotinsky, 2000; Lisman and Zhabotinsky, 2001; Miller et al., 2005). Consistent with this, genetic deletion of Thr286 blocks LTP and memory (Giese et al., 1998). More recent work shows that interfering with CaMKII during the maintenance phase of LTP can reverse LTP (Sanhueza et al., 2007). There is thus considerable evidence that CaMKII is critical for LTP, though other kinases may also have important roles (Pastalkova et al., 2006).

Less progress has been made in understanding the maintenance of LTD. In one class of models (Lisman, 1989), LTD is viewed as a reversal of LTP and occurs because CaMKII is dephosphorylated. However, while there is evidence that reversal of CaMKII occurs during depotentiation of experimentally induced LTP (Huang et al., 2001; Kang-Park et al., 2003), it now seems clear that LTD is fundamentally different from depotentiation (Zhuo et al., 1999). The first indication of this came from the finding that a phosphatase inhibitor reversed LTD when applied after LTD induction, but has no effect when applied under basal conditions (Mulkey et al., 1993). These results suggest that LTD alters the balance of phosphatase and kinase activities in favor of the phosphatase. Direct biochemical assays of phosphatase activity indicate that LTD induces a transient activation of PP1 and a persistent activation of the PP2A (Thiels et al., 1998). These reactions lead to changes in GluR phosphorylation (Lee et al., 2003). Importantly, the dephosphorylation of Ser845 during LTD is not the opposite of the phosphorylation changes that occur during LTP, which involves the phosphorylation of a 

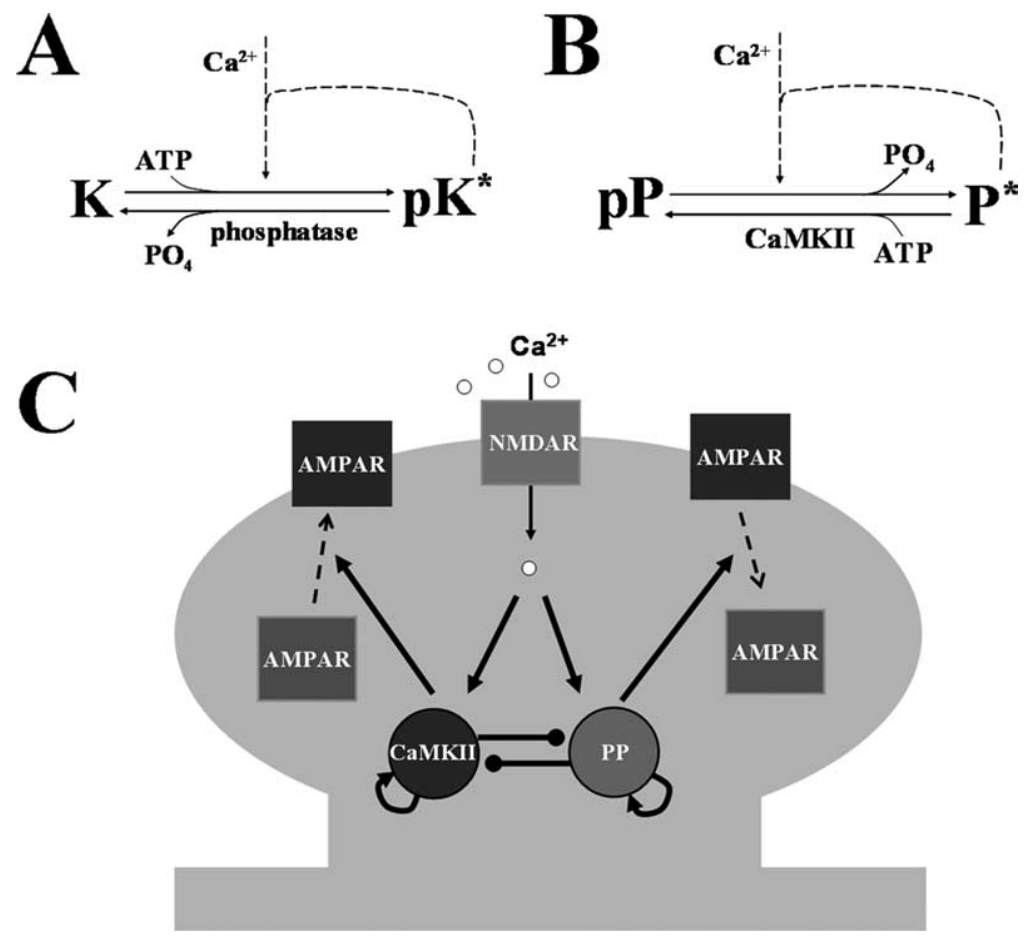

Figure 1. Simplified model. $\boldsymbol{A}$, Biochemical reactions of CaMKII. The kinase activity is turned on by $\mathrm{Ca}^{2+} / \mathrm{cal}_{\text {lmodulin stimu- }}$ lation or autocatalytic phosphorylation. $\boldsymbol{B}$, Biochemical reaction for phosphatase. The phosphatase activity is turned on by dephosphorylation, which is produced either by $\mathrm{Ca}^{2+}$ stimulation or autocatalytic dephosphorylation. K and P indicate kinase and phosphatase respectively; pK and pP are the phosphorylated forms of enzymes; ${ }^{*}$ indicates active enzyme. C, Simplified reaction scheme. Gray shading represents spine structure. Pointed and circled arrows indicate the activation and the inhibition pathways, respectively. $\mathrm{Ca}^{2+}$ influx through NMDAR can activate $\mathrm{CaMKII}(\mathrm{K})$ and protein phosphatase $(\mathrm{P})$ in a manner that depends on the concentration of $\mathrm{Ca}^{2+}$. CaMKIl and phosphatase can self-activate themselves and inhibit each other. Active kinase and phosphatase control AMPAR insertion and removal, respectively.

Ser831 by CaMKII (Lee et al., 2000). Together, these results leave little doubt that LTD is not simply a reversal of LTP but rather involves a different set of reactions. We have therefore sought to understand how known properties of PP2A might lead to its persistent activation during LTD and, more generally, how a tristable biochemical system might be formed that could account for both LTP and LTD.

\section{Materials and Methods}

CaMKII switch. In the model, $\mathrm{Ca}^{2+}$ influx through NMDAR activates both kinase $(\mathrm{K})$ and protein phosphatase $(\mathrm{P})$, which have different $\mathrm{I}_{\mathrm{C} 50}$ for $\mathrm{Ca}^{2+}$. The reaction scheme for CaMKII is described in Figure $1 \mathrm{~A} . \mathrm{K}$ and $\mathrm{pK}$ are the unphosphorylated and phosphorylated forms, and the ${ }^{*}$ indicates active enzyme. The kinase activity is turned on by phosphorylation, which is produced either by $\mathrm{Ca}^{2+}$ stimulation or autocatalytic phosphorylation. The distinction between inter and intramolecular autocatalytic reactions is not considered here to simplify the problem. Phosphatase (PP2A) inactivates $\mathrm{pK}$ by dephosphorylation. Assuming that enzymes kinetics follows Michealis-Menten scheme, the rate equation is expressed as

$$
\begin{aligned}
\frac{d}{d t} p K=k_{1} \frac{K}{K_{m 1}+K} p K-k_{2} \frac{p K}{K_{m 2}+p K}\left(P+P_{0}\right) & +k_{3} K_{0} \\
& +k_{4} \frac{C a^{4}}{K_{m}^{4}+C a^{4}} K
\end{aligned}
$$

where terms in the right-hand side are, in order, for autophosphorylation of kinase, dephosphorylation by phosphatase, basal kinase activity and $\mathrm{Ca}^{2+}$-dependent phosphorylation. $k_{1}, k_{2}, k_{3}$ and $k_{4}$ are rate constants for each reaction. $K_{\mathrm{m} 1}, K_{\mathrm{m} 2}$, and $K_{\mathrm{m}}$ are equilibrium constants for kinase, phosphatase and $\mathrm{Ca}^{2+}$, respectively. In the last term, the Hill coefficient of 4 is adapted from considering 4 binding of $\mathrm{Ca}^{2+}$ to activate CaMKII; calmodulin (CaM) has effectively two binding sites of $\mathrm{Ca}^{2+}$ and two $\mathrm{Ca}^{2+} / \mathrm{CaM}$ are required to initiate phosphorylation of CaMKII (De Koninck and Schulman, 1998). $K_{0}$ and $P_{0}$ indicate the basal concentration of active kinase and phosphatase, respectively. The resting $\mathrm{Ca}^{2+}$ concentration is set to $0.1 \mu \mathrm{M}$.

The total amount of $K$ and $P$ are conserved:

$$
\begin{aligned}
& K_{\text {tot }}=K+p K \\
& P_{\text {tot }}=P+p P .
\end{aligned}
$$

Phosphatase switch. The reaction scheme for phosphatase is described in Figure $1 B$ (see Results, Background: experimental findings regarding PP2A control for the reactions assumed). pP is phosphorylated (inactive) form. The activity of phosphatase is turned on by dephosphorylation either from $\mathrm{Ca}^{2+}$-dependent reaction pathways or from autocatalytic intermolecular dephosphorylation. pK inactivates phosphatase by phosphorylation. Then, the rate equation is as follows:

$$
\begin{aligned}
\frac{d}{d t} P= & k_{11} \frac{p P}{K_{m 11}+p P} P-k_{12} \frac{P}{K_{m 12}+P}(K \\
& \left.+K_{0}\right)+k_{13} P_{0}+k_{14} \frac{C a^{3}}{K_{m}^{3}+C a^{3}} p P,
\end{aligned}
$$

where terms on the right-hand side indicate, in order, autodephosphorylation of phosphatase, phosphorylation by kinase (CaMKII or other constitutive kinases) and $\mathrm{Ca}^{2+}$-dependent dephosphorylation. $k_{11}, k_{12}, k_{13}$ and $k_{14}$ are rate constants for each reaction. $K_{\mathrm{m} 11}$ and $K_{\mathrm{m} 12}$ are equilibrium constants for kinase and phosphatase. The Hill coefficient of 3 in the last term in Equation 4 describes the $\mathrm{Ca}^{2+}$-dependence of the dephosphorylation of the phosphatase. This may occur by several different pathways. To the extent that calcineurin is involved, the Hill coefficient is 3 (Stemmer and Klee, 1994). PP2A can also be activated via two $\mathrm{Ca}^{2+}$ bound to the PP2A regulatory subunit, B"/PR72 (Ahn et al., 2007), and, thus, the Hill coefficient is 2. Our simulations did not reveal strong differences depending on whether the Hill coefficient is 2 or 3 .

AMPAR trafficking. We have taken a simplified view of AMPAR trafficking to provide a readout of the kinase and phosphatase switches. AMPAR is inserted to the synaptic membrane with the rate constant $k_{21}$ and removed with rate constant $\mathrm{k}_{22}$. The reaction scheme for AMPAR is described as follows:

$$
\mathrm{A}_{\text {int }} \underset{k_{22}}{\stackrel{k_{21}}{\rightleftarrows}} \mathrm{A} .
$$

Then the rate equation is

$$
\frac{d}{d t} A=k_{21} A_{\text {int }}-k_{22} A,
$$

where $A$ and $A_{\text {int }}$ indicate the AMPAR on the synaptic membrane and the internalized AMPAR. The total AMPAR is assumed to be conserved on the time scale of these simulations.

$$
A_{\text {tot }}=A+A_{\text {int }} .
$$

Since the activities of kinase and phosphatase are thought to be key players in the insertion and the removal of AMPAR, $k_{21}$ and $k_{22}$ are 
dependent on their activities. There is considerable biological complexity to this control involving multiple phosphorylation sites on GluR1 and stargazin (Barria et al., 1997; Tomita et al., 2005); as a first approximation, $k_{21}$ and $k_{22}$ are assumed to be proportional to the concentration of active kinase and phosphatase, respectively.

$$
\begin{aligned}
& k_{21}=c_{1} p K+c_{3} \\
& k_{22}=c_{2} P+c_{4},
\end{aligned}
$$

where $c_{1}$ and $c_{2}$ are scaling factors (or proportional constants), $c_{3}$ and $c_{4}$ are rate constants for the processes independent of kinase and phosphatase activities. We stipulate that the excitatory postsynaptic potential (EPSP) is proportional to the amount of AMPAR on the synaptic membrane.

Parameters of the model are listed in supplemental Table S1, available at www.jneurosci.org as supplemental material. One of the key requirements for tristability is the correct balance between autocatalytic activities and mutual inhibitions of enzymes. Maximal kinase and phosphatase activities had to be at the same general level; too weak or too strong mutual inhibitions abolished tristability (see supplemental materials, available at www.jneurosci.org). Parameters in Equations 1 and 2 were adjusted to balance each switch to achieve tristability. Other parameter values did not change the qualitative features of the system (i.e., tristability). Therefore, other parameter values were chosen to reproduce basic aspects of LTP and LTD and then used consistently for all calculations. The numerical simulations were done with the fourth-order RungeKutta method using Matlab (version 7.0, The MathWorks).

\section{Results}

Background: experimental findings regarding PP2A control

The key idea we wish to introduce is that biochemical properties of PP2A could allow this enzyme to form a phosphatase switch that could underlie the maintenance of LTD. In this section, we briefly review relevant biochemical evidence.

An important observation regarding the regulation of PP2A is that it has autocatalytic dephosphorylation properties analogous to the autocatalytic kinase activity of CaMKII. The first to report the intermolecular self-activating mechanism of PP2A, called autodephosphorylation, was (Chen et al., 1992). The PP2A holoenzyme is composed of 3 subunits: the structural subunit (A), the regulatory subunit $(B)$, and the catalytic subunit $(C)$. Various combinations of $\mathrm{A}, \mathrm{B}$, and $\mathrm{C}$ subunits and multiple phosphorylation sites make it possible for PP2A to control multiple substrates in a finely tuned manner (Colbran, 2004). Chen et al. (1992) witnessed that the limited phosphate incorporation onto Tyr307 of the catalytic subunit of PP2A by tyrosine kinase p $60^{\mathrm{v}-\mathrm{src}}$ was maximized by the application of phosphatase inhibitor. They proposed that an autocatalytic activity of PP2A reverses the incorporation of phosphate by further showing thioATP (the phosphate thioester form is resistant to dephosphorylation) instead of ATP, gave rise to the maximal incorporation. Importantly, similar autodephosphorylation properties of PP2A were found at the Ser/Thr sites phosphorylated by CaMKII. Specifically, phosphorylation of B' $\alpha$ subunit, a regulatory $\mathrm{B}$ subunit of $\mathrm{PP} 2 \mathrm{~A}$, can undergo autodesphosphorylation (Fukunaga et al., 2000).

Two types of results demonstrate that interactions of PP2A and CaMKII lead to functional regulation of each other. The first is the ability of PP2A to dephosphorylate CaMKII (Fukunaga et al., 2000). Dephosphorylation of CaMKII leads to a loss of its $\mathrm{Ca}^{2+}$-independent activity (Miller and Kennedy, 1986). Most importantly, from the viewpoint of the switch model we will develop, CaMKII can phosphorylate and thereby inactivate PP2A (Fukunaga et al., 2000).

LTD is initiated by $\mathrm{Ca}^{2+}$ entry through NMDA channels and this entry is necessary for the activation of PP2A (Thiels et al., 1998). Many potential enzymatic pathways may couple this $\mathrm{Ca}^{2+}$ entry to PP2A. For instance, PP2A can be activated by $\mathrm{Ca}^{2+}$ through striatin (Moreno et al., 2000; Benoist et al., 2006) and/or $\mathrm{B}^{\prime \prime} /$ PR72 (Ahn et al., 2007). Striatin is a $\mathrm{Ca}^{2+} /$ calmodulinbinding protein associated with dendritic spines and PSDs (Moreno et al., 2000; Benoist et al., 2006). It physically binds to PP2A and regulates PP2A activity in a $\mathrm{Ca}^{2+} /$ calmodulin dependent manner. B"/PR72 is a regulatory B subunit of PP2A which has $\mathrm{Ca}^{2+}$ binding sites and is enriched in the striatum and hippocampus (Ahn et al., 2007). Still another possibility is that $\mathrm{Ca}^{2+}$ can activate PP2A via PKC-dependent pathways (Zhang et al., 2007). Finally, it is possible that PP2A is activated when it is dephosphorylated by calcineurin (PP2B) or PP1. The latter is activated during LTD by a calcineurin-I1 pathway (Mulkey et al., 1994). Given the lack of information that establishes the relative roles of these pathways, we have modeled a single $\mathrm{Ca}^{2+}$. dependent pathway for the activation of PP2A; this can be considered the lumped action of the known $\mathrm{Ca}^{2+}$-dependent pathways.

Although there has been consideration of the interplay of CaMKII and PP2A during LTP (Kikuchi et al., 2003), the importance of these reactions in the maintenance of LTD has not been previously considered. In the next section we show that the autocatalytic properties of PP2A could form the basis of a phosphatase switch that underlies LTD maintenance and that is compatible with a kinase switch that underlies LTP.

\section{Tristability of the synapse}

CaMKII and PP2A have autocatalytic properties and inhibit each other. These key reactions are schematized in Figure 1C. Thus, the system is such that both kinase and phosphatase are driven by autocatalytic enzyme activities that provide positive feedback to their activation. However, there is also mutual inhibition that prevents strong simultaneous activation of both enzymes. As we will show through simulation, these reactions can yield three stable states.

Theoretical and experimental studies suggest that the intracellular $\mathrm{Ca}^{2+}$ level determines whether LTP or LTD is induced (Lisman, 1989; Neveu and Zucker, 1996); a moderate level of $\mathrm{Ca}^{2+}$ drives the system to the LTD state, whereas a high level drives the system to the LTP state. Following previous models and experimental data (Stemmer and Klee, 1994; De Koninck and Schulman, 1998; Lisman and Zhabotinsky, 2001; Miller et al., 2005), we assume that initiation of CaMKII autophosphorylation requires a much higher $\mathrm{Ca}^{2+}$ level than the initiation of phosphatase activation. As we will show, this leads to different states after different levels of $\mathrm{Ca}^{2+}$ stimulation.

The results of simulations of the model described in Materials and Methods are shown in Figure 2. The LTP simulation results are illustrated as a black trace in Figure $2 \mathrm{~A}$. Starting from the initial basal state, a high $\mathrm{Ca}^{2+}$ pulse ( $4 \mu \mathrm{M}$ for $2 \mathrm{~s}$; gray area) drove the EPSP magnitude to the potentiated state, which was sustained even after $\mathrm{Ca}^{2+}$ removal. The change of phosphatase and kinase activities is shown in the left panel of Figure $2 B$. Both enzymes were nearly inactive in the basal state. During high $\mathrm{Ca}^{2+}$ elevation, the kinase became strongly activated. After the end of the induction period, $\mathrm{Ca}^{2+}$ returned to basal levels, but the kinase stayed in the on-state. The high kinase activity suppressed phosphatase activity below baseline levels (Fig. 2 B, inset; LTP graph), consistent with experimental results (Fukunaga et al., 2000).

Fig. $2 \mathrm{~A}$ (left) shows how the basal state is affected by moderate $\mathrm{Ca}^{2+}$ elevation $(2.2 \mu \mathrm{M}$ for $2 \mathrm{~s})$; as shown in the red trace, LTD is 
A
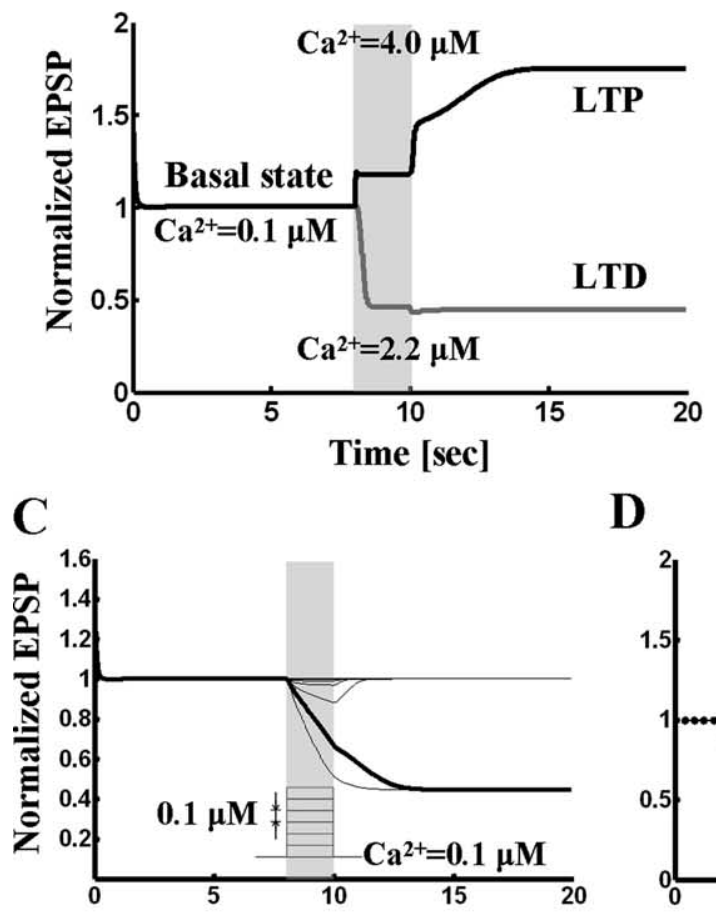

Time [sec]

D
B
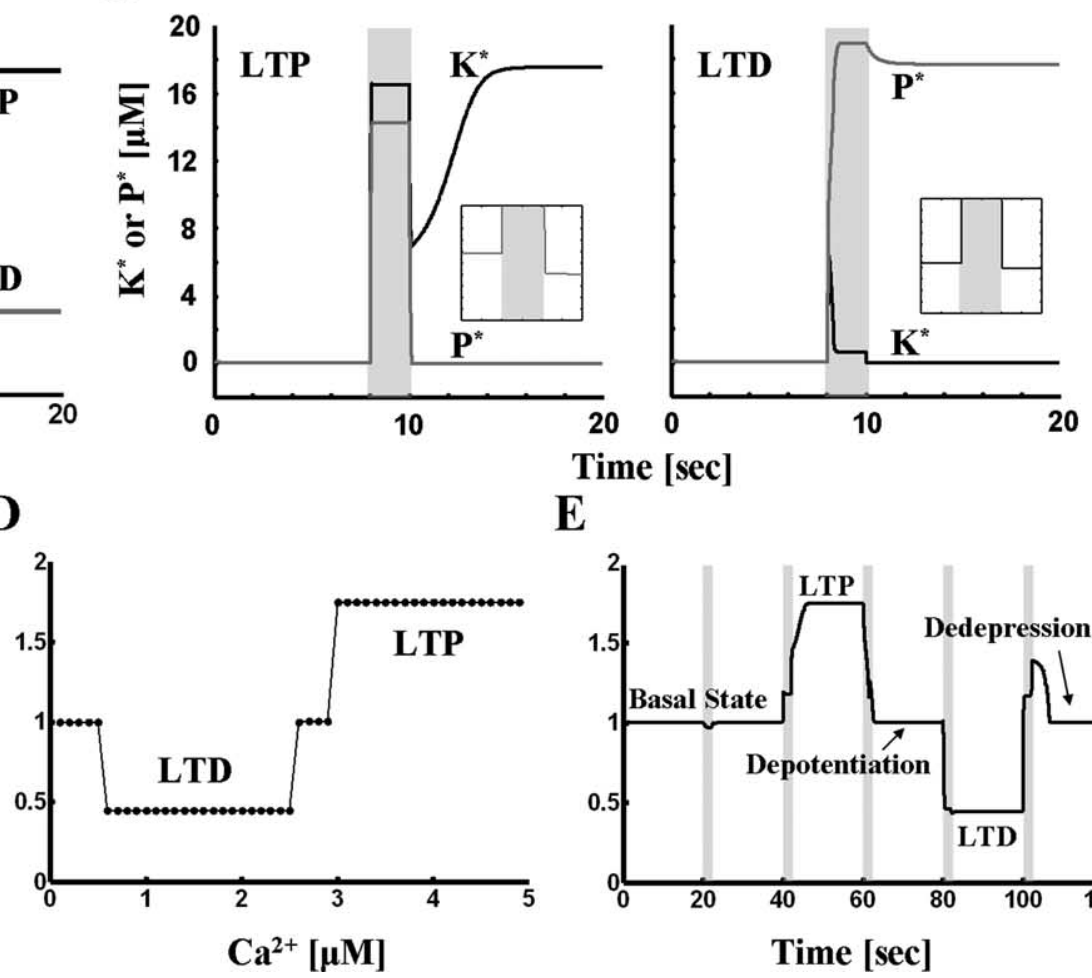

E

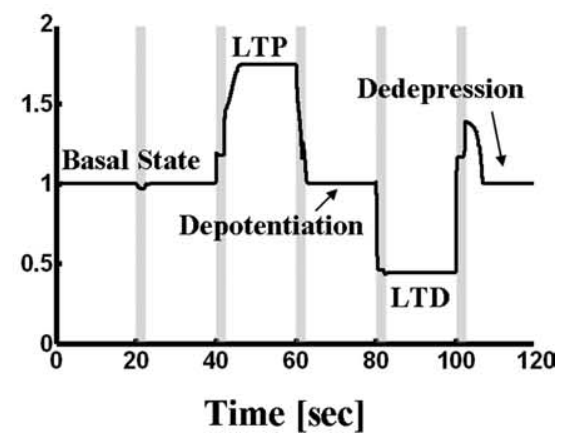

Figure 2. Coupled kinase and phosphatase switches can produce tristability. $A$, Simulation results for coupled switch model. The basal state undergoes LTP in response to high concentration (4 $\mu \mathrm{m}$ for $2 \mathrm{~s})$ of $\mathrm{Ca}^{2+}$. The basal state goes to LTD state in response to moderate $\mathrm{Ca}^{2+}$ elevation $(2.2 \mu \mathrm{m}$ for $2 \mathrm{~s}) . \boldsymbol{B}$, Enzyme activities during LTP and LTD. Left panel, Kinase and phosphatase activities during LTP. $K^{*}$ and $P^{*}$ denote the concentration of active kinase and phosphatase respectively. Before $\mathrm{Ca}^{2+}$ stimulation, both kinase and phosphatase are nearly inactive. During high level of $\mathrm{Ca}^{2+}$ application, kinase activity is dominant. After $\mathrm{Ca}^{2+}$ removal, active kinase represses the phosphatase activity below its initial level (see inset). Right panel, Kinase and phosphatase activities during LTD. Phosphatase is activated by $\mathrm{Ca}^{2+}$ pulse of $1 \mu \mathrm{m}$ for $2 \mathrm{~s}$ and stays in the active state after $\mathrm{Ca}^{2+}$ is removed. During maintenance of LTD, active phosphatase represses kinase activity (see inset). C, Robustness of basal state to perturbation. Different levels of $\mathrm{Ca}^{2+}$ pulses (step size, $0.1 \mu \mathrm{m}$ for $2 \mathrm{~s}$ ) are applied on top of basal concentration $(0.1 \mu \mathrm{m})$, and responses are traced. Basal state is robust to the $\mathrm{Ca}^{2+}$ concentration less than or equal to $0.5 \mu \mathrm{m}$. Concentrations $>0.6 \mu \mathrm{m}$ evoke LTD (red trace). D, Dependence of sign of synaptic modification on $\mathrm{Ca}^{2+}$ level during a pulse (note similarity to BCM curve). $E$, Reversals of LTP and LTD. The basal state which is robust to small perturbation (the first stimulation: $0.4 \mu \mathrm{m} \mathrm{Ca}^{2+}$ for $2 \mathrm{~s}$ ) undergoes LTP by the second stimulation (4 $\mu \mathrm{m}$ for $2 \mathrm{~s}$ ). The third stimulation ( $2.2 \mu \mathrm{m}$ for $1.4 \mathrm{~s}$ ) reverses the potentiated state back to basal level (depotentiation). The fourth ( $2.2 \mu \mathrm{m}$ for $2 \mathrm{~s}$ ) and the fifth ( $2.93 \mu \mathrm{m}$ for $2 \mathrm{~s}$ ) stimulations induce and reverse LTD, respectively (dedepression).

induced. The right panel of Figure $2 B$ shows how enzyme activities are affected by LTD. Both enzymes were nearly inactive in the initial basal state. During the moderate $\mathrm{Ca}^{2+}$ elevation, the phosphatase activity became dominant because it is more sensitive to $\mathrm{Ca}^{2+}$ than the kinase. After $\mathrm{Ca}^{2+}$ returned to the resting level, the phosphatase stayed in the on-state and suppressed the basal kinase activity (Fig. $2 \mathrm{~B}$, inset, right panel).

We next tested whether these three states are indeed stable. To be considered a stable state, it must be resistant to small perturbations. First, the basal state was tested. Different levels of $\mathrm{Ca}^{2+}$ pulses (step size, $0.1 \mu \mathrm{M}$ for $2 \mathrm{~s}$ ) were applied on top of the resting $\mathrm{Ca}^{2+}$ concentration $(0.1 \mu \mathrm{M})$ and responses were plotted (Fig. $2 C)$. The basal state returned to its original state after termination of small $\mathrm{Ca}^{2+}$ perturbations ranging from $0.1-0.5 \mu \mathrm{M}$. To test the stability of LTP and LTD states, similar small perturbations were applied. Again, both LTP and LTD states returned to their original states at the end of the perturbations (data not shown). Together, these results demonstrate that the basal state can undergo LTP or LTD depending upon the $\mathrm{Ca}^{2+}$ stimulation, and that each of the states is resistant to small perturbations. The system as whole is thus tristable. The results account for three experimental observations: the activation of CaMKII and inactivation of PP2A during LTP and the activation of PP2A during LTD (Fukunaga et al., 1993, 2000; Thiels et al., 1998).

To further characterize the transitions between states, we sys- tematically varied the concentration of $\mathrm{Ca}^{2+}$ pulses. For $\mathrm{Ca}^{2+}$ concentrations between 0.1 and $0.5 \mu \mathrm{M}$, the system stayed in basal state. In the range between 0.6 and $2.5 \mu \mathrm{M}$, the basal state underwent LTD while it underwent LTP in the $\mathrm{Ca}^{2+}$ concentration $>3.0 \mu \mathrm{M}$. Between 2.6 and $2.9 \mu \mathrm{M}$ of $\mathrm{Ca}^{2+}$, neither LTP nor LTD was inducible. Thus, a curve similar to the BCM curve (Bienenstock et al., 1982) is generated by plotting the final level of EPSP against $\mathrm{Ca}^{2+}$ concentration (Fig. 2D).

The reversal of LTP and LTD has been reported in experimental studies (Muller et al., 1995; Huang et al., 2001). The reverse of LTP and LTD is called depotentiation and dedepression, respectively. We tested whether such reversal could occur in our model. As shown in Figure 2E, this was the case. After LTP was induced from the basal state by high $\mathrm{Ca}^{2+}$ elevation ( $4 \mu \mathrm{M}$ for $2 \mathrm{~s}$ ), a moderate level of $\mathrm{Ca}^{2+}(2.2 \mu \mathrm{M}$ for $1.4 \mathrm{~s})$ reversed the potentiated state back to basal level (depotentiation). Stimulation with moderate $\mathrm{Ca}^{2+}(2.2 \mu \mathrm{M}$ for $2 \mathrm{~s})$ induced LTD, after which dedepression by high $\mathrm{Ca}^{2+}(2.93 \mu \mathrm{M}$ for $2 \mathrm{~s})$ reversed it (dedepression).

\section{Phase space analysis}

To understand the dynamics of the model graphically, nullclines at different $\mathrm{Ca}^{2+}$ concentrations (basal level, LTP induction and LTD induction) were plotted (Fig. 3). At resting $\mathrm{Ca}^{2+}$ level, nullclines (Fig. 3, left panel) intersected at five points; three were stable points (filled circles at top left, bottom left and bottom 


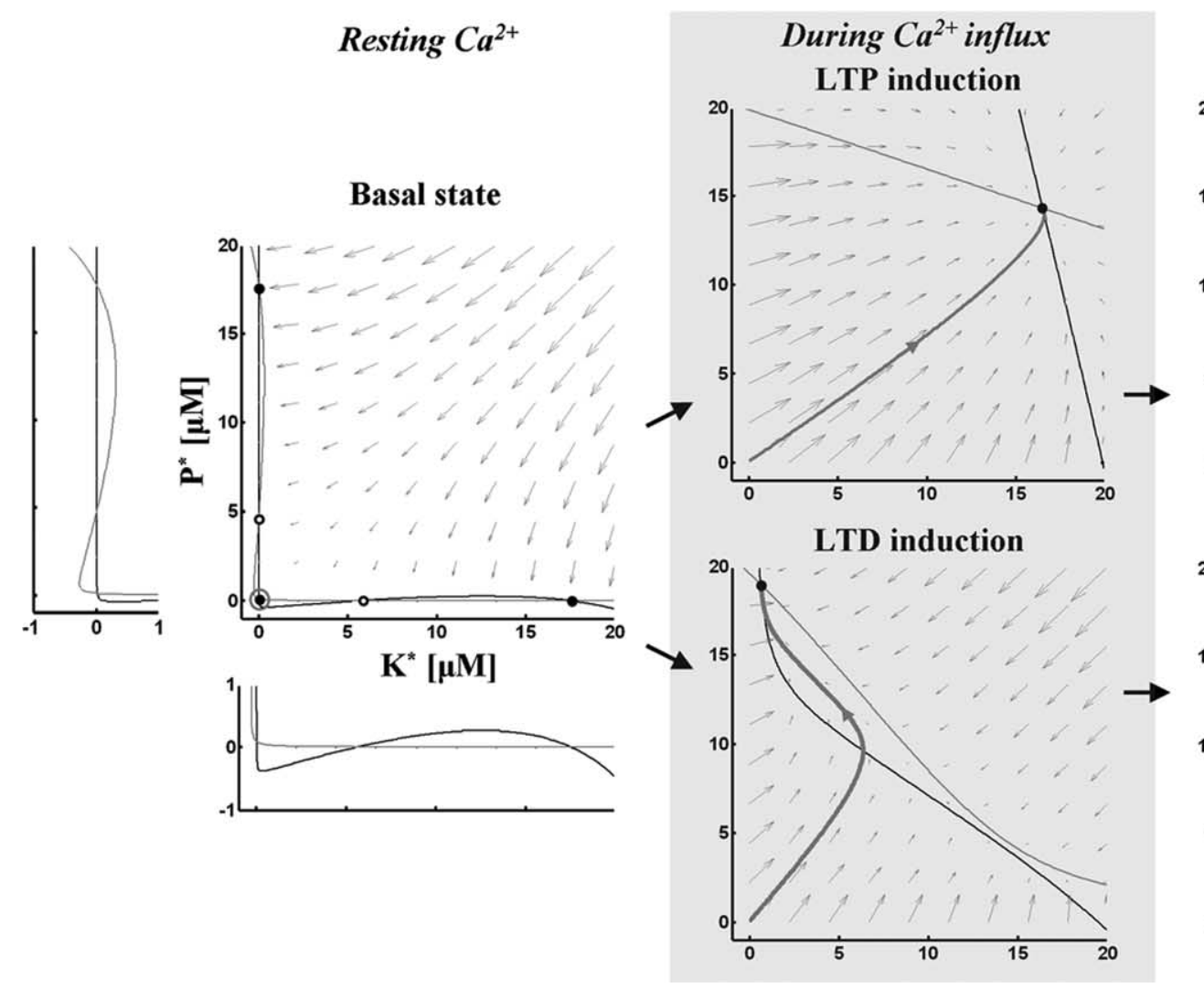

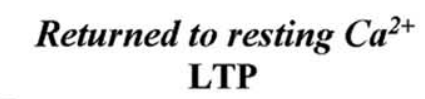
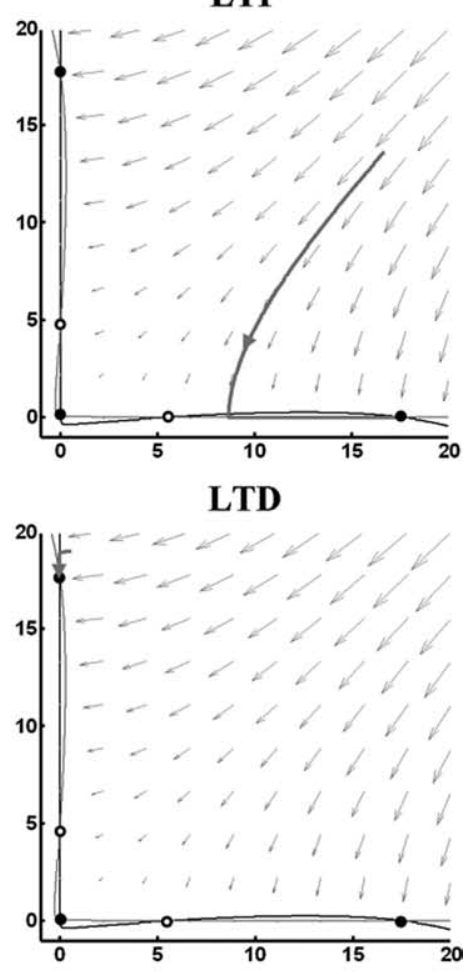

Figure 3. The dynamics of the model in phase space. Left panel, Black and gray curves indicate nullclines for kinase and phosphatase respectively. The vector field is indicated by gray arrows. Nullclines at resting $\mathrm{Ca}^{2+}$ concentration $(0.1 \mu \mathrm{m})$ create five steady-states. Open and filled circles denote unstable and stable steady-states, respectively. Gray open circle indicates the basal state. $\mathrm{K}^{*}$ and $\mathrm{P}^{*}$ denote the concentration of active kinase and phosphatase respectively. Insets, The areas where nullclines intersect are magnified. Middle panels, Nullclines during Ca ${ }^{2+}$ influx. Top, During LTP induction, the $\mathrm{Ca}^{2+}$ elevation ( $4 \mu \mathrm{m}$ for $2 \mathrm{~s}$ ) deforms nullclines to create one stable state. Bottom, During LTD induction, the moderate level of Ca ${ }^{2+}$ elevation ( $2.2 \mu \mathrm{m}$ for $2 \mathrm{~s}$ ) deforms nullclines to create one stable state. The basal state becomes unstable and moves to the closest stable state (gray trace). Right panels, Nullclines after Ca ${ }^{2+}$ removal. Nullclines form three stable states again in the resting $\mathrm{Ca}^{2+}$ concentration. The state moves to the closest stable state, LTP (gray trace, top) or LTD (gray trace, bottom).

right) and two were unstable (open circles). Small arrows indicate how the system moves to one of the stable points from any arbitrary point. In the basal state, the system was stable at the bottom left point. During LTP induction, the $\mathrm{Ca}^{2+}$ elevation deformed the nullclines to create only one stable state. After $\mathrm{Ca}^{2+}$ removal, nullclines formed three stable states again, but the system stayed in the bottom right stable point (LTP) (Fig. 3, top right panel). During LTD induction, the moderate level of $\mathrm{Ca}^{2+}$ elevation deformed nullclines to create one stable state. After $\mathrm{Ca}^{2+}$ removal, nullclines formed three stable states again and the system moved to top left stable point (LTD) (Fig. 3, bottom right panel). Phase space analysis for depotentiation and dedepression is described in supplemental materials, available at www. jneurosci.org as supplemental material.

\section{Discussion}

Although there has been substantial investigation of how a bistable kinase switch could account for a stable transition between the basal and LTP states, there has been relatively little experimental or theoretical work on the process that maintains LTD. The simulations presented show that biochemical mechanisms of PP2A, notably its autodephosphorylation properties, allow it to function as a bistable switch that could underlie LTD. We have further shown that the phosphatase and kinase switches can be coupled, giving rise to a tristable system. Transitions between states are triggered by moderate and high $\mathrm{Ca}^{2+}$ elevations that occur during LTD and LTP respectively (Lisman, 1989; Neveu and Zucker, 1996). Moreover, the model reproduces the BCM function (Bienenstock et al., 1982).

The proposed mechanism of persistent phosphatase activation is based on the principle of autocatalysis, specifically autodephosphorylation, for which there is direct experimental support. Thus, during LTD, PP2A is largely dephosphorylated and active; any PP2A that is phosphorylated by basal CaMKII activity is rapidly dephosphorylated by the remaining unphosphorylated PP2A, thereby maintaining a stable LTD state. It can be seen that the underlying principle is similar to that of the CaMKII switch responsible for LTP in which any site dephosphorylated by basal phosphatase activity is rephosphorylated by the remaining phosphorylated CaMKII.

In an earlier model of bidirectional modification, a single storage variable, the phosphorylation of CaMKII, could be bidirectionally modified, thereby accounting for LTP and LTD (Lisman, 1989). However, as reviewed in the Introduction, such a model does not account for the very different kinds of biochemical reactions observed during LTP and LTD. The current model can account for these observations because there are two interacting molecular switches, a kinase switch and a phosphatase switch. These switches are both stable and therefore can account for the maintenance of LTP and LTD. Other theoretical models have succeeded in describing how different levels of $\mathrm{Ca}^{2+}$ trigger LTP or LTD, but have avoided the issue of how LTP and LTD are maintained, focusing only 
on the induction processes (Migliore et al., 1995; Castellani et al., 2001, 2005; Zhabotinsky et al., 2006).

\section{Limitations of the model}

Several limitations of our model should be noted. Both LTP and LTD are synapse-specific modifications and it is therefore important to specify how the underlying biochemistry is localized to a particular synapse. In the case of the CaMKII, the localization of CaMKII in the PSD makes it likely that the CaMKII switch is actually localized in the PSD (Strack et al., 1997; Chen et al., 2005; Mullasseril et al., 2007). PP2A is present both in the cytoplasm and the PSD, but the current model is not explicit regarding the localization.

A second limitation stems from uncertainty about how $\mathrm{Ca}^{2+}$ activates PP2A during LTD induction. The calcineurin-I1-PP1 cascade could lead to PP2A dephosphorylation and thus activation (see review in Results), genetic deletion of forms of calcineurin either has no effect on LTD (Zhuo et al., 1999) or produces only a partial block of LTD (Zeng et al., 2001). Perhaps most likely is that multiple pathways are involved. As mentioned in Results, PP2A can be activated by $\mathrm{Ca}^{2+}$ through striatin, a $\mathrm{Ca}^{2+} /$ calmodulin-binding protein associated with dendritic spines and PSDs (Moreno et al., 2000; Benoist et al., 2006) and/or $\mathrm{B}^{\prime \prime} / \mathrm{PR} 72$, a regulatory B subunit of PP2A which has $\mathrm{Ca}^{2+}$ binding sites and is enriched in the striatum and hippocampus (Ahn et al., 2007). Still another possibility is that $\mathrm{Ca}^{2+}$ can activate PP2A via PKC-dependent pathways (Zhang et al., 2007). There is also uncertainty about the targets of PP2A. LTD affects the balance of PKA and PP1 activities and thereby leads to a reduction of Ser845 on GluR1. How this might be controlled by PP2A activity remains unclear. We speculate that PP2A may dephosphorylate cofilin, thereby leading to a reduction of F-actin and the associated AKAP-PKA complex which alter the balance of PKA and PP1. A final limitation is that the proposed model is applicable only to early LTD because the protein synthesis required for late LTD (Sajikumar and Frey, 2003) is not taken into consideration.

\section{Data accounted for and predictions}

Our model suggests a mechanism for the persistent activation PP2A induced by LTD induction (Thiels et al., 1998). No previous explanation of this persistence has been proposed. A critical prediction of our model is that PP2A activation should be necessary for LTD induction and maintenance. After finishing our simulations (Nicholls et al., 2008) reported that this is the case; specific inhibition of PP2A blocks NMDAR-dependent LTD without affecting LTP, depotentiation and mGluR LTD. Whether PP2A is also required for LTD maintenance (this is tested by inhibiting the enzyme after induction) has not been tested. Further experiments may test the role of autodephosphorylation, the reaction that provides the positive feedback that maintains PP2A activity. Mutant of this site to a pseudophosphorylated form this should block LTD; mutation to a nonphosphorylatable form should lock the synapse in the LTD state.

\section{References}

Ahn JH, Sung JY, McAvoy T, Nishi A, Janssens V, Goris J, Greengard P, Nairn AC (2007) The B"/PR72 subunit mediates Ca2+-dependent dephosphorylation of DARPP-32 by protein phosphatase 2A. Proc Natl Acad Sci U S A 104:9876-9881.

Barria A, Muller D, Derkach V, Griffith LC, Soderling TR (1997) Regulatory phosphorylation of AMPA-type glutamate receptors by CaM-KII during long-term potentiation. Science 276:2042-2045.

Benoist M, Gaillard S, Castets F (2006) The striatin family: a new signaling platform in dendritic spines. J Physiol Paris 99:146-153.
Bienenstock EL, Cooper LN, Munro PW (1982) Theory for the development of neuron selectivity: orientation specificity and binocular interaction in visual cortex. J Neurosci 2:32-48.

Castellani GC, Quinlan EM, Cooper LN, Shouval HZ (2001) A biophysical model of bidirectional synaptic plasticity: dependence on AMPA and NMDA receptors. Proc Natl Acad Sci U S A 98:12772-12777.

Castellani GC, Quinlan EM, Bersani F, Cooper LN, Shouval HZ (2005) A model of bidirectional synaptic plasticity: from signaling network to channel conductance. Learn Mem 12:423-432.

Chen J, Martin BL, Brautigan DL (1992) Regulation of protein serinethreonine phosphatase type-2A by tyrosine phosphorylation. Science 257:1261-1264.

Chen X, Vinade L, Leapman RD, Petersen JD, Nakagawa T, Phillips TM, Sheng M, Reese TS (2005) Mass of the postsynaptic density and enumeration of three key molecules. Proc Natl Acad Sci USA 102:11551-11556.

Colbran RJ (2004) Protein phosphatases and calcium/calmodulindependent protein kinase II-dependent synaptic plasticity. J Neurosci 24:8404-8409.

De Koninck P, Schulman H (1998) Sensitivity of CaM kinase II to the frequency of Ca2 + oscillations. Science 279:227-230.

Fukunaga K, Stoppini L, Miyamoto E, Muller D (1993) Long-term potentiation is associated with an increased activity of $\mathrm{Ca} 2+/$ calmodulindependent protein kinase II. J Bio Chem 268:7863-7867.

Fukunaga K, Muller D, Miyamoto E (1995) Increased phosphorylation of $\mathrm{Ca} 2+/$ calmodulin-dependent protein kinase II and its endogenous substrates in the induction of long-term potentiation. J Biol Chem 270:6119-6124.

Fukunaga K, Muller D, Ohmitsu M, Bakó E, DePaoli-Roach AA, Miyamoto E (2000) Decreased protein phosphatase 2A activity in hippocampal longterm potentiation. J Neurochem 74:807-817.

Giese KP, Fedorov NB, Filipkowski RK, Silva AJ (1998) Autophosphorylation at Thr286 of the alpha calcium-calmodulin kinase II in LTP and learning. Science 279:870-873.

Huang CC, Liang YC, Hsu KS (2001) Characterization of the mechanism underlying the reversal of long term potentiation by low frequency stimulation at hippocampal CA1 synapses. J Biol Chem 276:48108-48117.

Kang-Park MH, Sarda MA, Jones KH, Moore SD, Shenolikar S, Clark S, Wilson WA (2003) Protein phosphatases mediate depotentiation induced by high-intensity theta-burst stimulation. J Neurophysiol 89:684-690.

Kikuchi S, Fujimoto K, Kitagawa N, Fuchikawa T, Abe M, Oka K, Takei K, Tomita M (2003) Kinetic simulation of signal transduction system in hippocampal long-term potentiation with dynamic modeling of protein phosphatase 2A. Neural Netw 16:1389-1398.

Lee HK, Barbarosie M, Kameyama K, Bear MF, Huganir RL (2000) Regulation of distinct AMPA receptor phosphorylation sites during bidirectional synaptic plasticity. Nature 405:955-959.

Lee HK, Takamiya K, Han JS, Man H, Kim CH, Rumbaugh G, Yu S, Ding L, He C, Petralia RS, Wenthold RJ, Gallagher M, Huganir RL (2003) Phosphorylation of the AMPA receptor GluR1 subunit is required for synaptic plasticity and retention of spatial memory. Cell 112:631-643.

Lisman J (1989) A mechanism for the Hebb and the anti-Hebb processes underlying learning and memory. Proc Natl Acad Sci USA 86:9574-9578.

Lisman JE (1985) A mechanism for memory storage insensitive to molecular turnover: a bistable autophosphorylating kinase. Proc Natl Acad Sci U S A 82:3055-3057.

Lisman JE, Zhabotinsky AM (2001) A model of synaptic memory: a CaMKII/PP1 switch that potentiates transmission by organizing an AMPA receptor anchoring assembly. Neuron 31:191-201.

Matsuzaki M, Honkura N, Ellis-Davies GC, Kasai H (2004) Structural basis of long-term potentiation in single dendritic spines. Nature 429:761-766.

Migliore M, Alicata F, Ayala GF (1995) A model for long-term potentiation and depression. J Comput Neurosci 2:335-343.

Miller P, Zhabotinsky AM, Lisman JE, Wang XJ (2005) The stability of a stochastic CaMKII switch: dependence on the number of enzyme molecules and protein turnover. PLoS Biol 3:e107.

Miller SG, Kennedy MB (1986) Regulation of brain type II Ca2+/ calmodulin-dependent protein kinase by autophosphorylation: a Ca2+triggered molecular switch. Cell 44:861-870.

Moreno CS, Park S, Nelson K, Ashby D, Hubalek F, Lane WS, Pallas DC 
(2000) WD40 repeat proteins striatin and S/G(2) nuclear autoantigen are members of a novel family of calmodulin-binding proteins that associate with protein phosphatase 2A. J Biol Chem 275:5257-5263.

Mulkey RM, Herron CE, Malenka RC (1993) An essential role for protein phosphatases in hippocampal long-term depression. Science 261:1051-1055.

Mulkey RM, Endo S, Shenolikar S, Malenka RC (1994) Involvement of a calcineurin/inhibitor-1 phosphatase cascade in hippocampal long-term depression. Nature 369:486-488.

Mullasseril P, Dosemeci A, Lisman JE, Griffith LC (2007) A structural mechanism for maintaining the 'on-state' of the CaMKII memory switch in the post-synaptic density. J Neurochem 103:357-364.

Muller D, Hefft S, Figurov A (1995) Heterosynaptic interactions between LTP and LTD in CA1 hippocampal slices. Neuron 14:599-605.

Neveu D, Zucker RS (1996) Long-lasting potentiation and depression without presynaptic activity. J Neurophysiol 75:2157-2160.

Nicholls RE, Alarcon JM, Malleret G, Carroll RC, Grody M, Vronskaya S, Kandel ER (2008) Transgenic mice lacking NMDAR-dependent LTD exhibit deficits in behavioral flexibility. Neuron 58:104-117.

O'Connor DH, Wittenberg GM, Wang SS (2005) Graded bidirectional synaptic plasticity is composed of switch-like unitary events. Proc Natl Acad Sci U S A 102:9679-9684.

Pastalkova E, Serrano P, Pinkhasova D, Wallace E, Fenton AA, Sacktor TC (2006) Storage of spatial information by the maintenance mechanism of LTP. Science 313:1141-1144.

Petersen CC, Malenka RC, Nicoll RA, Hopfield JJ (1998) All-or-none potentiation at CA3-CA1 synapses. Proc Natl Acad Sci U S A 95:4732-4737.

Sajikumar S, Frey JU (2003) Anisomycin inhibits the late maintenance of long-term depression in rat hippocampal slices in vitro. Neurosci Lett 338:147-150.

Sanhueza M, McIntyre CC, Lisman JE (2007) Reversal of synaptic memory by $\mathrm{Ca}^{2+} /$ calmodulin-dependent protein kinase II inhibitor. J Neurosci 27:5190-5199.
Sobczyk A, Svoboda K (2007) Activity-dependent plasticity of the NMDAreceptor fractional Ca2 + current. Neuron 53:17-24.

Stemmer PM, Klee CB (1994) Dual calcium ion regulation of calcineurin by calmodulin and calcineurin B. Biochemistry 33:6859-6866.

Strack S, Barban MA, Wadzinski BE, Colbran RJ (1997) Differential inactivation of postsynaptic density-associated and soluble Ca2+/calmodulindependent protein kinase II by protein phosphatases 1 and 2A. J Neurochem 68:2119-2128.

Thiels E, Norman ED, Barrionuevo G, Klann E (1998) Transient and persistent increases in protein phosphatase activity during long-term depression in the adult hippocampus in vivo. Neuroscience 86:1023-1029.

Tomita S, Stein V, Stocker TJ, Nicoll RA, Bredt DS (2005) Bidirectional synaptic plasticity regulated by phosphorylation of stargazin-like TARPs. Neuron 45:269-277.

Zeng H, Chattarji S, Barbarosie M, Rondi-Reig L, Philpot BD, Miyakawa T, Bear MF, Tonegawa S (2001) Forebrain-specific calcineurin knockout selectively impairs bidirectional synaptic plasticity and working/episodiclike memory. Cell 107:617-629.

Zhabotinsky AM (2000) Bistability in the $\mathrm{Ca}(2+) /$ calmodulin-dependent protein kinase-phosphatase system. Biophys J 79:2211-2221.

Zhabotinsky AM, Camp RN, Epstein IR, Lisman JE (2006) Role of the neurogranin concentrated in spines in the induction of long-term potentiation. J Neurosci 26:7337-7347.

Zhang D, Kanthasamy A, Yang Y, Anantharam V, Kanthasamy A (2007) Protein kinase $\mathrm{C}$ delta negatively regulates tyrosine hydroxylase activity and dopamine synthesis by enhancing protein phosphatase-2A activity in dopaminergic neurons. J Neurosci 27:5349-5362.

Zhou Q, Xiao M, Nicoll RA (2001) Contribution of cytoskeleton to the internalization of AMPA receptors. Proc Natl Acad Sci U S A 98:1261-1266.

Zhuo M, Zhang W, Son H, Mansuy I, Sobel RA, Seidman J, Kandel ER (1999) A selective role of calcineurin aalpha in synaptic depotentiation in hippocampus. Proc Natl Acad Sci U S A 96:4650-4655. 